\title{
On multicurve models for the term structure
}

\author{
Laura Morino* \\ Dipartimento di Matematica Pura ed Applicata \\ Università di Padova, Via Trieste 63, I-35121-Padova \\ e-mail: laura.morino88@gmail.com \\ Wolfgang J. Runggaldier \\ Dipartimento di Matematica Pura ed Applicata \\ Università di Padova, Via Trieste 63, I-35121-Padova \\ e-mail: runggal@math.unipd.it
}

\begin{abstract}
In the context of multi-curve modeling we consider a two-curve setup, with one curve for discounting (OIS swap curve) and one for generating future cash flows (LIBOR for a give tenor). Within this context we present an approach for the clean-valuation pricing of FRAs and CAPs (linear and nonlinear derivatives) with one of the main goals being also that of exhibiting an "adjustment factor" when passing from the one-curve to the two-curve setting. The model itself corresponds to short rate modeling where the short rate and a short rate spread are driven by affine factors; this allows for correlation between short rate and short rate spread as well as to exploit the convenient affine structure methodology. We briefly comment also on the calibration of the model parameters, including the correlation factor.
\end{abstract}

Mathematics Subject Classification : Primary 91G30; Secondary 91G20, $60 \mathrm{H} 30$.

Keywords : Multicurve models, affine factor models, interest rate derivatives, clean valuation, adjustment factors.

\section{Introduction}

In the wake of the big crisis one has witnessed a significant increase in the spreads between LIBORs of different tenors as well as the spread between a LIBOR and the discount curve (LIBOR-OIS). This has led to the construction of multicurve models where, typically, future cash flows are generated

\footnotetext{
${ }^{*}$ Present affiliation: Deloitte Consulting Srl., Milano.
} 
through curves associated to the underlying rates, but are discounted by another curve.

The majority of the models that have been considered reflects the usual classical distinction between

i) short rate models;

ii) HJM setup;

iii) BGM or LIBOR market models.

By analogy to credit risk we may call the first two categories of models as bottom-up models, while the third one could be classified as top-down. In addition, methodologies have appeared that are related to foreign exchange.

Here we consider only the first two setups. We begin by discussing some issues arising with the HJM methodology and concentrate then on short rate models. The third setup (top-down) is mainly present in work by F. Mercurio and co-authors (see e.g. [19], [20]), but also in other recent work such as [16. There are advantages and disadvantages with each setup. Among the possible advantages of short rate models is the fact that they lead more easily to a Markovian setting, which is convenient for various calculations (see [8]). On the other hand, one of the major advantages of HJM over a direct short rate modeling is that the model is automatically calibrated to the initial term structure. Short rate models in a multi-curve setup have already appeared in the literature, e.g. [18, [17, [11.

To present the basic ideas in a simple way, here we consider a twocurve model, namely with a curve for discounting and one for generating future cash flows. The choice of the discount curve is not unique; we follow the common choice of considering the OIS swap curve. For the risky cash flows without collateral we consider a single LIBOR (i.e. for a given tenor structure).

We present an approach for the pricing of some basic LIBOR-related derivatives, namely FRAs and CAPs (linear/nonlinear) and consider only clean valuation formulas, namely without counterparty risk. Although real pricing problems require a more global approach (see e.g. the discussions in [13], 14], 22], 6] as well as in recent work by D.Brigo and co-authors such as [21], 2]), clean valuation formulas are nevertheless useful for various reasons: as pointed out in [8, market quotes typically reflect prices of fully collateralized transactions so that clean price formulas may turn out to be sufficient for calibration also when using the model to compute possible value adjustments; furthermore (see [8]), TVA adjustments are often computed on top of clean prices. Concerning methodology, since our approach is of the bottom-up type that considers short rate modeling, we heavily exploit the advantages of an affine term structure. This is in contrast with topdown approaches, where (see [19], [20]) log-normal models are common (see 
however [16] and [15] for affine LIBOR models with general distributions in a multicurve context).

Traditionally, interest rates are defined to be coherent with the bond prices $p(t, T)$, which represent the expectation of the market concerning the future value of money. For the discrete compounding forward LIBORs, which we denote here by $L(t ; T, S)$, this leads to $(t<T<S)$

$$
L(t ; T, S)=\frac{1}{S-T}\left(\frac{p(t, T)}{p(t, S)}-1\right)
$$

which can also be justified as representing the fair value of the fixed rate in a FRA on the LIBOR. Since we consider only a single LIBOR that corresponds to a given tenor structure, we assume $S=T+\Delta$ (for tenor $\Delta$ ). In this way one obtains a single curve for the term structure. The actual LIBOR rates, which in what follows we shall denote by $\bar{L}(t ; T, T+\Delta)$, are determined by the LIBOR panel that takes into account various factors such as credit risk, liquidity, etc. (see the discussion in [11]). Following some of the recent literature, in particular [7] (see also [18), we keep the formal relationship (1.1) between LIBOR rates and bond prices, but replace the risk-free bond prices $p(t, T)$ by fictitious "risky" bond prices $\bar{p}(t, T)$ that are supposed to be affected by the same factors as the actual LIBORs and that, analogously to the risk-free bond prices, we define then as

$$
\bar{p}(t, T)=E^{Q}\left\{\exp \left[-\int_{t}^{T}\left(r_{u}+s_{u}\right) d u\right] \mid \mathcal{F}_{t}\right\}
$$

where $r_{t}$ is the classical short rate, whereas $s_{t}$ represents the short rate spread (hazard rate in case of only default risk). Notice that in this way the spread is introduced from the outset. Notice also that the fictitious bond prices $\bar{p}(t, T)$ are not actual prices.

Since in what follows we are interested in FRAs and CAPs that are based on the $T$-spot LIBOR $\bar{L}(t ; T, T+\Delta)$, we actually postulate the relationship (1.1) only at the inception time $t=T$. Our starting point is thus the following relationship

$$
\bar{L}(T ; T, T+\Delta)=\frac{1}{\Delta}\left(\frac{1}{\bar{p}(T, T+\Delta)}-1\right)
$$

where we have taken into account the fact that also for the "risky" bonds we have $\bar{p}(T, T)=1$.

In addition to the pricing of FRAs and CAPs in our two-curve setup, our major goal here is to derive a relationship between theoretically risk-free and actual FRAs (possibly also CAPs) thereby exhibiting an adjustment factor which plays a role analogous to that of the quanto adjustments in the pricing of cross-currency derivatives or the "multiplicative forward basis" in [1]. 


\section{The model}

\subsection{Preliminary considerations}

We start with some comments concerning HJM-like approaches to better motivate our short rate approach. Given the bond price processes $p(t, T)$ and $\bar{p}(t, T)$, in order to apply an HJM-approach, we need to introduce corresponding forward rate processes $f^{T}(t)$ and $\bar{f}^{T}(t)$ that lead to a forward rate spread expressed as $g^{T}(t):=\bar{f}^{T}(t)-f^{T}(t)$. One then also obtains corresponding short rates and a short rate spread, namely $r_{t}=f^{t}(t), \bar{r}_{t}=\bar{f}^{t}(t), s_{t}=g^{t}(t)=\bar{r}_{t}-r_{t}$. Notice that a consistent model should lead to $\bar{p}(t, T) \leq p(t, T)$, which implies $\bar{f}^{T}(t) \geq f^{T}(t)$ or, equivalently $g^{T}(t) \geq 0 \forall t<T \leq \bar{T}$, where $\bar{T}$ is a given maximal maturity.

An extensive study within the multicurve HJM approach has appeared in [7]. The driving random process is a Levy and a corresponding HJM drift condition is derived. Conditions are given for the non-negativity of rates and spreads; explicit formulas are obtained for various interest rate derivatives. What may not be fully satisfactory in [7] is that:

i) some difficulties arise when dealing not only with credit risk, but also other risks such as liquidity. In particular, when looking for a condition that corresponds to the defaultable HJM drift condition;

ii) a fictitious default has to be considered explicitly (with pre default bond prices).

The study in [7] is continued in the recent paper [8] with the main purpose of taking into account also counterparty risk and funding costs and of determining various valuation adjustments on top of the clean prices. The methodology in [8] is again based on an HJM approach, but with explicit ingredients for the induced short rate models in order to obtain a Markovian structure and to be able to actually perform the value adjustment calculations. In particular, the authors in 8 use a Levy Hull \& White extended Vasicek model for $r_{t}$ and introduce an additional factor that can be interpreted as representing a short rate spread. In this latter sense it becomes analogous to the approach to be presented here.

Another HJM-based approach, limited to default risk, appears in 3] with emphasis on obtaining Markovian models with state dependent volatilities. The driving processes are of the jump-diffusion type. The difficulties here appear to be given by the fact that, for convenient specifications of the volatilities, one obtains deterministic short rate spreads. For more general, stochastic volatilities the authors obtain only approximate Markovianity. These difficulties have been overcome in the subsequent paper [4, where the authors obtain finite-dimensional Markovian realizations also with stochastic spreads and, in addition, obtain a correlation structure between 
credit spread, interest rate and the stochastic volatility. When trying to extend their approach to a multi curve setting, beyond that implied by credit risk alone, there appear though some computational difficulties due to the stochastic volatility.

Before coming now to describing our short rate model, we recall some basics concerning FRAs. We start from the

Definition 2.1. A FRA (forward rate agreement) is an OTC derivative that allows the holder to lock in at $t<T$ the interest rate between the inception date $T$ and the maturity $T+\Delta$ at a fixed value $K$. At maturity $T+\Delta$, a payment based on $K$ is made and one based on $\bar{L}(T ; T, T+\Delta)$ is received.

We shall denote the value of the FRA at $t<T$ by $F R A^{T}(t, K)$. In our two-curve risky setup, the fair price of a FRA in $t<T$ with fixed rate $K$ and notional $N$ is

$$
\begin{aligned}
F R A^{T}(t, K) & =N \Delta p(t, T+\Delta) E^{T+\Delta}\left[\bar{L}(T ; T, T+\Delta)-K \mid \mathcal{F}_{t}\right] \\
& =N p(t, T+\Delta) E^{T+\Delta}\left[\frac{1}{\bar{p}(T, T+\Delta)}-(1+\Delta K) \mid \mathcal{F}_{t}\right]
\end{aligned}
$$

where $E^{T+\Delta}$ denotes expectation under the $(T+\Delta)$ - forward measure $Q^{T+\Delta}$. Notice that the simultaneous presence of $p(t, T+\Delta)$ and $\bar{p}(t, T+\Delta)$ does not allow for the convenient reduction of the formula to a simpler form as in the one-curve setup.

\subsection{Description of the model itself}

For the short-rate model approach we shall have to start by modeling directly the short rate $r_{t}$ and the short rate spread $s_{t}$ and we do it under the standard martingale measure $Q$ (to be calibrated to the market) for the risk-free money market account as numeraire. In order to account for a possible (negative) correlation between $r_{t}$ and $s_{t}$ we introduce a factor model: given three independent affine factor processes $\Psi_{t}^{i}, i=1,2,3$ let

$$
\left\{\begin{array}{l}
r_{t}=\Psi_{t}^{2}-\Psi_{t}^{1} \\
s_{t}=\kappa \Psi_{t}^{1}+\Psi_{t}^{3}
\end{array}\right.
$$

where $\kappa$ is a constant that measures the instantaneous correlation between $r_{t}$ and $s_{t}$ (negative correlation for $\kappa>0$ ). This setup could be generalized in various ways, in particular by using more factors to drive $s_{t}$. In view of the existing literature one could, instead of using an affine model structure as we do it here, consider e.g. ambit-type processes as presented in [5. Such a model, which is not of the semimartingale type, allows also for analytical computations and gives the possibility to take into account long-range dependence. Remaining within the pure credit risk setting where, see the comment after (1.2), the spread is given by the default intensity, some of the factors affecting the spread could be given a specific meaning as in [9] 
where, using an HJM-type approach, the authors consider a spread field process with one of the variables representing the rating of the issuer. The approach in [9] could possibly be generalized also tio the present setting.

A common approach to modeling the factors in an affine context is to assume them of the type of a square root diffusion. This guarantees positivity of the spread, but the negative correlation comes at the expense of possibly negative interest rates (even if only with small probability). With such a model, by passing to the $(T+\Delta)$-forward measure, one can compute the value of a FRA and of the fair fixed rate.

For various reasons, in particular in view of our main goal to obtain an adjustment factor, it is convenient to be able to have the same factor model for FRAs with different maturities. We therefore aim at performing the calculations under a single reference measure, namely the standard martingale measure $Q$. More precisely, for the factor processes we assume the following affine diffusions under $Q$ that are of the Vasicek type, namely

$$
\left\{\begin{array}{l}
d \Psi_{t}^{1}=\left(a^{1}-b^{1} \Psi_{t}^{1}\right) d t+\sigma^{1} d w_{t}^{1} \\
d \Psi_{t}^{i}=\left(a^{i}-b^{i} \Psi_{t}^{i}\right) d t+\sigma^{i} \sqrt{\Psi_{t}^{i}} d w_{t}^{i}, \quad i=2,3
\end{array}\right.
$$

where $a^{i}, b^{i}, \sigma^{i}$ are positive constants with $a^{i} \geq\left(\sigma^{i}\right)^{2} / 2$ for $i=2,3$, and $w_{t}^{i}$ independent Wiener processes. We have chosen a Vasicek-type model for simplicity, but the results below can be easily extended to the Hull \& White version of the Vasicek model. Notice that the factor $\Psi_{t}^{1}$ may take negative values implying that, not only $r_{t}$, but also $s_{t}$ may become negative (see however later under "comments on the main result"). Results completely analogous to those that we shall obtain here for the above pure diffusion model may be derived also for affine jump-diffusions at the sole expense of more complicated notation.

\section{Main result (FRAs)}

\subsection{Preliminary notions and results}

Recalling the expression for a FRA under the forward measure, namely

$$
F R A^{T}(t, K)=N p(t, T+\Delta) E^{T+\Delta}\left[\frac{1}{\bar{p}(T, T+\Delta)}-(1+\Delta K) \mid \mathcal{F}_{t}\right],
$$

one has that the crucial quantity to compute is

$$
\bar{\nu}_{t, T}:=E^{T+\Delta}\left[\frac{1}{\bar{p}(T, T+\Delta)} \mid \mathcal{F}_{t}\right]
$$


and that the fixed rate to make the FRA a fair contract at time $t$ is

$$
\bar{K}_{t}:=\frac{1}{\Delta}\left(\bar{\nu}_{t, T}-1\right)
$$

In the classical single curve case we have instead

$$
\nu_{t, T}:=E^{T+\Delta}\left[\frac{1}{p(T, T+\Delta)} \mid \mathcal{F}_{t}\right]=\frac{p(t, T)}{p(t, T+\Delta)}
$$

being $\frac{p(t, T)}{p(t, T+\Delta)}$ an $\mathcal{F}_{t}$-martingale under the $(T+\Delta)$ - forward measure. The fair fixed rate in the single curve case is then

$$
K_{t}=\frac{1}{\Delta}\left(\nu_{t, T}-1\right)=\frac{1}{\Delta}\left(\frac{p(t, T)}{p(t, T+\Delta)}-1\right)
$$

and notice that, in order to compute $K_{t}$, no interest rate model is needed (contrary to $\bar{K}_{t}$ ).

Due to the affine dynamics of $\Psi_{t}^{i}(i=1,2,3)$ under $Q$, we have for the risk-free bond

$$
\begin{aligned}
p(t, T) & =E^{Q}\left\{\exp \left[-\int_{t}^{T} r_{u} d u\right] \mid \mathcal{F}_{t}\right\}=E^{Q}\left\{\exp \left[\int_{t}^{T}\left(\Psi_{u}^{1}-\Psi_{u}^{2}\right) d u\right] \mid \mathcal{F}_{t}\right\} \\
& =\exp \left[A(t, T)-B^{1}(t, T) \Psi_{t}^{1}-B^{2}(t, T) \Psi_{t}^{2}\right]
\end{aligned}
$$

The coefficients satisfy

$$
\left\{\begin{array}{lll}
B_{t}^{1}-b^{1} B^{1}-1=0 & , & B^{1}(T, T)=0 \\
B_{t}^{2}-b^{2} B^{2}-\frac{\left(\sigma^{2}\right)^{2}}{2}\left(B^{2}\right)^{2}+1=0 & , & B^{2}(T, T)=0 \\
A_{t}=a^{1} B^{1}-\frac{\left(\sigma^{1}\right)^{2}}{2}\left(B^{1}\right)^{2}+a^{2} B^{2} & , & A(T, T)=0
\end{array}\right.
$$

leading, in particular, to

$$
B^{1}(t, T)=\frac{1}{b^{1}}\left(e^{-b^{1}(T-t)}-1\right) .
$$

For the risky bond we have instead

$$
\begin{aligned}
\bar{p}(t, T) & =E^{Q}\left\{\exp \left[-\int_{t}^{T}\left(r_{u}+s_{u}\right) d u\right] \mid \mathcal{F}_{t}\right\} \\
& =E^{Q}\left\{\exp \left[-\int_{t}^{T}\left((\kappa-1) \Psi_{u}^{1}+\Psi_{u}^{2}+\Psi_{u}^{3}\right) d u\right] \mid \mathcal{F}_{t}\right\} \\
& =\exp \left[\bar{A}(t, T)-\bar{B}^{1}(t, T) \Psi_{t}^{1}-\bar{B}^{2}(t, T) \Psi_{t}^{2}-\bar{B}^{3}(t, T) \Psi_{t}^{3}\right]
\end{aligned}
$$


This time the coefficients satisfy

$$
\left\{\begin{array}{lll}
\bar{B}_{t}^{1}-b^{1} \bar{B}^{1}+(\kappa-1)=0 & , & \bar{B}^{1}(T, T)=0 \\
\bar{B}_{t}^{2}-b^{2} \bar{B}^{2}-\frac{\left(\sigma^{2}\right)^{2}}{2}\left(\bar{B}^{2}\right)^{2}+1=0 & , \quad \bar{B}^{2}(T, T)=0 \\
\bar{B}_{t}^{3}-b^{3} \bar{B}^{3}-\frac{\left(\sigma^{3}\right)^{2}}{2}\left(\bar{B}^{3}\right)^{2}+1=0 & , \quad \bar{B}^{3}(T, T)=0 \\
\bar{A}_{t}=a^{1} \bar{B}^{1}-\frac{\left(\sigma^{1}\right)^{2}}{2}\left(\bar{B}^{1}\right)^{2}+a^{2} \bar{B}^{2}+a^{3} \bar{B}^{3} & , \quad \bar{A}(T, T)=0
\end{array}\right.
$$

leading, in particular, to

$$
\bar{B}^{1}(t, T)=\frac{1-\kappa}{b^{1}}\left(e^{-b^{1}(T-t)}-1\right)=(1-\kappa) B^{1}(t, T)
$$

From the above $1-$ st order equations it follows that

$$
\left\{\begin{aligned}
\bar{B}^{1}(t, T)= & (1-\kappa) B^{1}(t, T) \\
\bar{B}^{2}(t, T)= & B^{2}(t, T) \\
\bar{A}(t, T)= & A(t, T)-a^{1} \kappa \int_{t}^{T} B^{1}(u, T) d u \\
& +\frac{\left(\sigma^{1}\right)^{2}}{2} \kappa^{2} \int_{t}^{T}\left(B^{1}(u, T)\right)^{2} d u-\left(\sigma^{1}\right)^{2} \kappa \int_{t}^{T} B^{1}(u, T) d u \\
& -a^{3} \int_{t}^{T} \bar{B}^{3}(u, T) d u
\end{aligned}\right.
$$

Letting then

$$
\tilde{A}(t, T):=\bar{A}(t, T)-A(t, T)
$$

we obtain

$$
\begin{aligned}
\bar{p}(t, T)= & \exp \left[\bar{A}(t, T)-B^{1}(t, T) \Psi_{t}^{1}-B^{2}(t, T) \Psi_{t}^{2}\right. \\
& \left.-\bar{B}^{3}(t, T) \Psi_{t}^{3}+\kappa B^{1}(t, T) \Psi_{t}^{1}\right] \\
= & p(t, T) \exp \left[\tilde{A}(t, T)+\kappa B^{1}(t, T) \Psi_{t}^{1}-\bar{B}^{3}(t, T) \Psi_{t}^{3}\right]
\end{aligned}
$$

so that, putting for simplicity $\tilde{B}^{1}:=B^{1}(T, T+\Delta)$, one may write

$$
\frac{p(T, T+\Delta)}{\bar{p}(T, T+\Delta)}=\exp \left[-\tilde{A}(T, T+\Delta)-\kappa \tilde{B}^{1} \Psi_{T}^{1}+\bar{B}^{3}(T, T+\Delta) \Psi_{T}^{3}\right] .
$$

\subsection{The result itself}

We introduce the

Definition 3.1. We call adjustment factor the process

$$
A d_{t}^{T, \Delta}:=E^{Q}\left\{\frac{p(T, T+\Delta)}{\bar{p}(T, T+\Delta)} \mid \mathcal{F}_{t}\right\}
$$


and shall prove the following

Proposition 3.1. We have

$$
\bar{\nu}_{t, T}=\nu_{t, T} \cdot A d_{t}^{T, \Delta} \cdot \exp \left[\kappa \frac{\left(\sigma^{1}\right)^{2}}{2\left(b^{1}\right)^{3}}\left(1-e^{-b^{1} \Delta}\right)\left(1-e^{-b^{1}(T-t)}\right)^{2}\right]
$$

with two adjustment factors on the right, of which the first one can be expressed as

$$
\begin{aligned}
A d_{t}^{T, \Delta} & =e^{-\tilde{A}(T, T+\Delta)} E^{Q}\left\{e^{-\kappa \tilde{B}^{1} \Psi_{T}^{1}+\bar{B}^{3}(T, T+\Delta) \Psi_{T}^{3}} \mid \mathcal{F}_{t}\right\} \\
& :=A\left(\theta, \kappa, \Psi_{t}^{1}, \Psi_{t}^{3}\right)
\end{aligned}
$$

with $\theta:=\left(a^{i}, b^{i}, \sigma^{i}, i=1,2,3\right)$.

One may notice the analogy here with the multiplicative forward basis in $[1$.

As a consequence of the previous proposition we have the following relation between the fair value $\bar{K}_{t}$ of the fixed rate in an actual FRA and the fair value $K_{t}$ in a corresponding riskless one:

Corollary 3.1. The following relationship holds

$\bar{K}_{t}=\left(K_{t}+\frac{1}{\Delta}\right) \cdot A d_{t}^{T, \Delta} \cdot \exp \left[\kappa \frac{\left(\sigma^{1}\right)^{2}}{2\left(b^{1}\right)^{3}}\left(1-e^{-b^{1} \Delta}\right)\left(1-e^{-b^{1}(T-t)}\right)^{2}\right]-\frac{1}{\Delta}$

Notice that the factor given by the exponential is equal to 1 for zero correlation, i.e. for $(\kappa=0)$.

\subsection{Comments on the main result}

\subsubsection{Comments concerning the adjustment factors}

An easy intuitive interpretation of the main result can be obtained in the case of $\kappa=0$ (independence of $r_{t}$ and $s_{t}$ ): in this case we have $r_{t}+s_{t}>r_{t}$ implying $\bar{p}(T, T+\Delta)<p(T, T+\Delta)$ so that $A d_{t}^{T, \Delta} \geq 1$ (the exponential adjustment factor is equal to 1 ). As expected, from Proposition 3.1 and Corollary 3.1 it then follows that

$$
\bar{\nu}_{t, T} \geq \nu_{t, T} \quad, \quad \bar{K}_{t} \geq K_{t}
$$

To gain some intuition for the cases when $\kappa \neq 0$, let $\bar{p}^{\kappa}(t, T), \bar{\nu}_{t, T}^{\kappa}, A d_{t}^{T, \Delta, \kappa}$ denote the given quantities by stressing that the correlation parameter has value $\kappa$. Notice that $p(t, T)$ and thus also $\nu_{t, T}$ do not depend on $\kappa$. Consider then the case $\kappa>0$, which is the standard case implying negative correlation between $r_{t}$ and $s_{t}$. (The case $\kappa<0$ is analogous/dual). For illustrative purposes we distinguish between the two events $\left\{\Psi_{t}^{1}>0, \forall t \in[T, T+\right.$ 
$\Delta]\},\left\{\Psi_{t}^{1}<0, \forall t \in[T, T+\Delta]\right\}$ where the latter occurs only with small probability (in reality, $\Psi_{t}^{1}$ will be positive for certain values of $t$ and negative for the remaining ones).

On $\left\{\Psi_{t}^{1}>0, t \in[T, T+\Delta]\right\}$ we now have

$$
\begin{aligned}
& \bar{p}^{\kappa}(T, T+\Delta)<\bar{p}^{0}(T, T+\Delta) \\
& \Rightarrow \bar{\nu}_{t, T}^{\kappa}>\bar{\nu}_{t, T}^{0} \quad \Rightarrow \quad \bar{\nu}_{t, T}^{\kappa} / \nu_{t, T}>\bar{\nu}_{t, T}^{0} / \nu_{t, T}
\end{aligned}
$$

Recalling then

$$
\bar{\nu}_{t, T}^{\kappa}=\nu_{t, T} \cdot A d_{t}^{T, \Delta, \kappa} \cdot \exp \left[\kappa \frac{\left(\sigma^{1}\right)^{2}}{2\left(b^{1}\right)^{3}}\left(1-e^{-b^{1} \Delta}\right)\left(1-e^{-b^{1}(T-t)}\right)^{2}\right]
$$

the last inequality in (3.21) can be seen to be in line with the fact that, in this case, in (3.22) the exponential factor is $>1$ and $A d_{t}^{T, \Delta, \kappa}>A d_{t}^{T, \Delta, 0}$ (recall Definition 3.1).

On the other hand, on $\left\{\Psi_{t}^{1}<0, t \in[T, T+\Delta]\right\}$, we have

$$
\bar{p}^{\kappa}(T, T+\Delta)>\bar{p}^{0}(T, T+\Delta) \quad \Rightarrow \quad \bar{\nu}_{t, T}^{\kappa} / \nu_{t, T}<\bar{\nu}_{t, T}^{0} / \nu_{t, T}
$$

This inequality can be seen to be in line with the fact that, here, $A d_{t}^{T, \Delta, \kappa}<$ $A d_{t}^{T, \Delta, 0}$, but the exponential factor is still $>1$. This can nevertheless be explained by noticing that, in this case, $r_{t}$ is relatively large and $r_{t}+s_{t}$ is closer to $r_{t}$ (may be even $<r_{t}$ ). This implies a push of $\bar{\nu}_{t, T}^{\kappa} / \nu_{t, T}$ towards smaller values than in the previous case.

\subsubsection{Comments concerning the use of the results for calibration}

For what concerns calibration of our model to FRA and other available market data, notice that the coefficients $a^{1}, a^{2}, b^{1}, b^{2}, \sigma^{1}, \sigma^{2}$ can be calibrated in the usual way on the basis of the observations of default-free bonds $p(t, T)$ (if we had a Hull\& White extension of our Vasicek-type model (2.3) then also for this model the calibration could be performed as in the standard case). To calibrate $a^{3}, b^{3}, \sigma^{3}$, notice that, contrary to $p(t, T)$, the "risky" bonds $\bar{p}(t, T)$ are not observable (relation (1.3) does not imply a unique inverse relationship to determine $\bar{p}(t, T)$ from observations of the LIBORs). One can however observe $K_{t}=\frac{1}{\Delta}\left(\frac{p(t, T)}{p(t, T+\Delta)}-1\right)$ as well as the "risky" FRA rate $\bar{K}_{t}$. Recalling then Corollary 3.1 and the fact that $A d_{t}^{T, \Delta}=A\left(\theta, \kappa, \Psi_{t}^{1}, \Psi_{t}^{3}\right)$, notice that, having calibrated $a^{i}, b^{i}, \sigma^{i}(i=1,2)$, from the observations of $K_{t}$ and $\bar{K}_{t}$ one could thus calibrate $a^{3}, b^{3}, \sigma^{3}$ as well as $\kappa$. If there is a way to determine directly $A d_{t}^{T, \Delta}$ (e.g. by observing the FRA rates for uncorrelated $r_{t}$ and $s_{t}$ ), then the relationship between $K_{t}$ and $\bar{K}_{t}$ as expressed in Corollary 3.1 would allow to calibrate separately $\kappa$. We furthermore recall that, as pointed out in [8], calibration of clean prices is sufficient also when using the model to compute possible value adjustments. 


\subsection{Proof of the main result}

Since the quantities of interest, namely $\bar{\nu}_{t, T}$ and $\nu_{t, T}$ were defined under the forward measure (see (3.2) and (3.4)), as a first step we perform a change from the forward measure $Q^{T+\Delta}$ to the standard martingale measure $Q$. To this effect, putting $b_{t}:=\exp \left[\int_{0}^{t} r_{u} d u\right]$, the density process for changing from $Q$ to $Q^{T+\Delta}$ is $L_{t}=\frac{p(t, T+\Delta)}{p(0, T+\Delta) b_{t}}$. We can thus write

$$
\begin{aligned}
\bar{\nu}_{t, T} & =E^{T+\Delta}\left\{\frac{1}{\bar{p}(T, T+\Delta)} \mid \mathcal{F}_{t}\right\}=L_{t}^{-1} E^{Q}\left\{\frac{L_{T+\Delta}}{\bar{p}(T, T+\Delta)} \mid \mathcal{F}_{t}\right\} \\
& =\frac{1}{p(t, T+\Delta)} E^{Q}\left\{\exp \left[-\int_{t}^{T} r_{u} d u\right] \frac{p(T, T+\Delta)}{\bar{p}(T, T+\Delta)} \mid \mathcal{F}_{t}\right\}
\end{aligned}
$$

Recalling the expression for $p(T, T+\Delta) / \bar{p}(T, T+\Delta)$ (see (3.15) ) this becomes

$$
\begin{gathered}
\bar{\nu}_{t, T}=\frac{1}{p(t, T+\Delta)} E^{Q}\left\{e^{-\int_{t}^{T} r_{u} d u}\right. \\
\left.\cdot \exp \left[-\tilde{A}(T, T+\Delta)-\kappa \tilde{B}^{1} \Psi_{T}^{1}+\bar{B}^{3}(T, T+\Delta) \Psi_{T}^{3}\right] \mid \mathcal{F}_{t}\right\} \\
=\frac{1}{p(t, T+\Delta)} \exp [-\tilde{A}(T, T+\Delta)] E^{Q}\left\{e^{\bar{B}^{3}(T, T+\Delta) \Psi_{T}^{3}} \mid \mathcal{F}_{t}\right\} \\
\cdot E^{Q}\left\{e^{-\int_{t}^{T}\left(-\Psi_{u}^{1}+\Psi_{u}^{2}\right) d u} e^{-\kappa \tilde{B}^{1} \Psi_{T}^{1}} \mid \mathcal{F}_{t}\right\}
\end{gathered}
$$

To proceed, consider the process $F_{t}$ given by the last factor in (3.25), namely

$$
F_{t}:=E^{Q}\left\{e^{-\int_{t}^{T}\left(-\Psi_{u}^{1}+\Psi_{u}^{2}\right) d u} e^{-\kappa \tilde{B}^{1} \Psi_{T}^{1}} \mid \mathcal{F}_{t}\right\}
$$

Due to the affine dynamics of $\Psi_{t}^{i}, i=1,2$, and the independence of $\Psi_{t}^{1}$ and $\Psi_{t}^{2}$, we may write

$$
\begin{aligned}
& F_{t}:=E^{Q}\left\{e^{\int_{t}^{T} \Psi_{u}^{1} d u} e^{-\kappa \tilde{B}^{1} \Psi_{T}^{1}} \mid \mathcal{F}_{t}\right\} E^{Q}\left\{e^{-\int_{t}^{T} \Psi_{u}^{2} d u} \mid \mathcal{F}_{t}\right\} \\
& =\exp \left[\alpha^{1}(t, T)-\beta^{1}(t, T) \Psi_{t}^{1}\right] \exp \left[\alpha^{2}(t, T)-\beta^{2}(t, T) \Psi_{t}^{2}\right]
\end{aligned}
$$

where the coefficients satisfy

$$
\begin{cases}\beta_{t}^{1}-b^{1} \beta^{1}-1=0 & , \beta^{1}(T, T)=\kappa \tilde{B}^{1} \\ \beta_{t}^{2}-b^{2} \beta^{2}-\frac{\left(\sigma^{2}\right)^{2}}{2}\left(\beta^{2}\right)^{2}+1=0 & , \beta^{2}(T, T)=0 \\ \alpha_{t}^{1}=-\frac{\left(\sigma^{1}\right)^{2}}{2}\left(\beta^{1}\right)^{2}+a^{1} \beta^{1} & , \alpha^{1}(T, T)=0 \\ \alpha_{t}^{2}=a^{2} \beta^{2} & , \alpha^{2}(T, T)=0\end{cases}
$$


Recalling also (3.6)-( $(3.8)$, the solutions of the system (3.28) can be expressed as

$$
\left\{\begin{aligned}
\beta^{1}(t, T)= & \frac{1}{b^{1}}\left[\left(b^{1} \kappa \tilde{B}^{1}+1\right) e^{-b^{1}(T-t)}-1\right]=B^{1}(t, T)+\kappa \tilde{B}^{1} e^{-b^{1}(T-t)} \\
\beta^{2}(t, T)= & B^{2}(t, T) \\
\alpha^{1}(t, T)= & \frac{\left(\sigma^{1}\right)^{2}}{2} \int_{t}^{T}\left(\beta^{1}(u, T)\right)^{2} d u-a^{1} \int_{t}^{T} \beta^{1}(u, T) d u \\
= & \frac{\left(\sigma^{1}\right)^{2}}{2} \int_{t}^{T}\left(B^{1}(u, T)\right)^{2} d u-a^{1} \int_{t}^{T} B^{1}(u, T) d u \\
& +\frac{\left(\sigma^{1}\right)^{2}}{2}\left(\kappa \tilde{B}^{1}\right)^{2} \int_{t}^{T} e^{-2 b^{1}(T-u)} d u \\
& +\kappa \tilde{B}^{1}\left(\sigma^{1}\right)^{2} \int_{t}^{T} B^{1}(u, T) e^{-b^{1}(T-u)} d u-a^{1} \kappa \tilde{B}^{1} \int_{t}^{T} e^{-b^{1}(T-u)} d u \\
\alpha^{2}(t, T)= & -a^{2} \int_{t}^{T} B^{2}(u, T) d u
\end{aligned}\right.
$$

Consequently

$$
\begin{gathered}
F_{t}=\exp \left[\frac{\left(\sigma^{1}\right)^{2}}{2} \int_{t}^{T}\left(B^{1}(u, T)\right)^{2} d u-a^{1} \int_{t}^{T} B^{1}(u, T) d u\right. \\
\left.-a^{2} \int_{t}^{T} B^{2}(u, T) d u-B^{1}(t, T) \Psi_{t}^{1}-B^{2}(t, T) \Psi_{t}^{2}\right] \\
\cdot \exp \left[\frac{\left(\sigma^{1}\right)^{2}}{2}\left(\kappa \tilde{B}^{1}\right)^{2} \int_{t}^{T} e^{-2 b^{1}(T-u)} d u-a^{1} \kappa \tilde{B}^{1} \int_{t}^{T} e^{-b^{1}(T-u)} d u\right. \\
\left.-\kappa \tilde{B}^{1} e^{-b^{1}(T-t)} \Psi_{t}^{1}\right] \\
\cdot \exp \left[\kappa \tilde{B}^{1}\left(\sigma^{1}\right)^{2} \int_{t}^{T} B^{1}(u, T) e^{-b^{1}(T-u)} d u\right] \\
=p(t, T) \cdot \exp \left[\frac{\left(\sigma^{1}\right)^{2}}{2}\left(\kappa \tilde{B}^{1}\right)^{2} \int_{t}^{T} e^{-2 b^{1}(T-u)} d u-a^{1} \kappa \tilde{B}^{1} \int_{t}^{T} e^{-b^{1}(T-u)} d u\right. \\
\cdot \exp \left[\kappa \tilde{B}^{1}\left(\sigma^{1}\right)^{2} \int_{t}^{T} B^{1}(u, T) e^{-b^{1}(T-u)} d u\right]
\end{gathered}
$$

On the other hand, recalling (3.15), one obtains

$$
\begin{aligned}
E^{Q} & \left\{\frac{p(T, T+\Delta)}{\bar{p}(T, T+\Delta)} \mid \mathcal{F}_{t}\right\} \\
& =e^{-\tilde{A}(T, T+\Delta)} E^{Q}\left\{e^{\bar{B}^{3}(T, T+\Delta) \Psi_{T}^{3}} \mid \mathcal{F}_{t}\right\} E^{Q}\left\{e^{-\kappa \tilde{B}^{1} \Psi_{T}^{1}} \mid \mathcal{F}_{t}\right\}
\end{aligned}
$$

where, due to the affine dynamics of $\Psi_{t}^{1}$, we may write

$$
E^{Q}\left\{e^{-\kappa \tilde{B}^{1} \Psi_{T}^{1}} \mid \mathcal{F}_{t}\right\}=\exp \left[\bar{\alpha}(t, T)-\bar{\beta}(t, T) \Psi_{t}^{1}\right]
$$

with $\bar{\alpha}(\cdot)$ and $\bar{\beta}(\cdot)$ satisfying

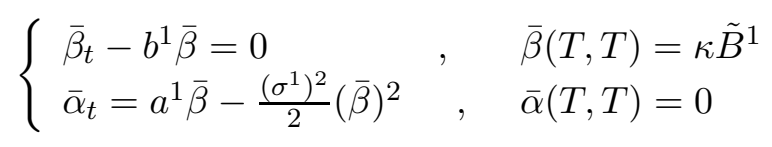


so that

$$
\begin{aligned}
& \bar{\beta}(t, T)=\kappa \tilde{B}^{1} e^{-b^{1}(T-t)} \\
& \bar{\alpha}(t, T)=-a^{1} \kappa \tilde{B}^{1} \int_{t}^{T} e^{-b^{1}(T-u)} d u+\frac{\left(\sigma^{1}\right)^{2}}{2}\left(\kappa \tilde{B}^{1}\right)^{2} \int_{t}^{T} e^{-2 b^{1}(T-u)} d u
\end{aligned}
$$

and,consequently,

$$
\begin{aligned}
& E^{Q}\left\{e^{\left.-\kappa \tilde{B}^{1} \Psi_{T}^{1} \mid \mathcal{F}_{t}\right\}=\exp \left[-\kappa \tilde{B}^{1} e^{-b^{1}(T-t)} \Psi_{t}^{1}\right]}\right. \\
& \quad \exp \left[-a^{1} \kappa \tilde{B}^{1} \int_{t}^{T} e^{-b^{1}(T-u)} d u+\frac{\left(\sigma^{1}\right)^{2}}{2}\left(\kappa \tilde{B}^{1}\right)^{2} \int_{t}^{T} e^{-2 b^{1}(T-u)} d u\right]
\end{aligned}
$$

Combining (3.25) with (3.30) as well as with (3.31) together with (3.35), we obtain

$$
\begin{gathered}
\bar{\nu}_{t, T}=\frac{1}{p(t, T+\Delta)} \exp [-\tilde{A}(T, T+\Delta)] E^{Q}\left\{e^{\bar{B}^{3}(T, T+\Delta) \Psi_{T}^{3}} \mid \mathcal{F}_{t}\right\} \cdot F_{t} \\
=\frac{p(t, T)}{p(t, T+\Delta)} E^{Q}\left\{\frac{p(T, T+\Delta)}{\bar{p}(T, T+\Delta)} \mid \mathcal{F}_{t}\right\} \\
\cdot \exp \left[\kappa\left(\sigma^{1}\right)^{2} \tilde{B}^{1} \int_{t}^{T} B^{1}(u, T) e^{-b^{1}(T-u)} d u\right] .
\end{gathered}
$$

The result then follows noticing that

$$
\tilde{B}^{1} \int_{t}^{T} B^{1}(u, T) e^{-b^{1}(T-u)} d u=\frac{1}{2\left(b^{1}\right)^{3}}\left(1-e^{-b^{1} \Delta}\right)\left(1-e^{-b^{1}(T-t)}\right)^{2} .
$$

\section{Aspects of CAP pricing}

\subsection{Preliminary comments}

This part is related to work in progress, but we want nevertheless to present some ideas on how our results obtained for FRAs (linear derivatives) can be extended to nonlinear derivatives. To discuss a specific case, we concentrate here on the pricing of a single Caplet, with strike $K$, maturity $T$ on the spot LIBOR for the period $[T, T+\Delta]$. Using the forward measure $Q^{T+\Delta}$, its price in $t<T$ is then given by

$$
\begin{aligned}
\operatorname{Capl}^{T, \Delta}(t) & =\Delta p(t, T+\Delta) E^{T+\Delta}\left\{(\bar{L}(T ; T, T+\Delta)-K)^{+} \mid \mathcal{F}_{t}\right\} \\
& =p(t, T+\Delta) E^{T+\Delta}\left\{\left(\frac{1}{\bar{p}(T, T+\Delta)}-\tilde{K}\right)^{+} \mid \mathcal{F}_{t}\right\}
\end{aligned}
$$

with $\tilde{K}:=1+\Delta K$.

As model, we may use the same "risky" short rate model as for the FRAs that we may consider as already calibrated (for the standard martingale measure $Q$ ). It may thus suffice to derive just a pricing algorithm that need 
not also be used for calibration. It remains however desirable to obtain also here an "adjustment factor".

The aim, pursued in the case of the FRAs, of performing the calculations under the same measure $Q$ leads here to some difficulties and so we stick to forward measures.

\subsection{A possible pricing methodology}

For the pricing, in the forward measure, we may use Fourier transform methods as in [7] and [8] thereby representing the claim as

$$
\left(e^{X}-\bar{K}\right)^{+} \quad \text { with } \quad X:=-\log \bar{p}(T, T+\Delta)
$$

We then need only to compute the moment generating function of $X$, which is a linear combination of the factors (this computation is feasible thanks to the affine structure) and use the Fourier transform of $f(x)=\left(e^{x}-\bar{K}\right)^{+}$, which is well-known.

Notice that one could possibly also apply a Gram-Charlier expansion as in [18].

With the Fourier transform method the price in $t=0$ of the Caplet can then be obtained in the form (see [8])

$$
\operatorname{Capl}(0, T, T+\Delta)=\frac{p(0, T+\Delta)}{2 \pi} \int \frac{\tilde{K}^{1-i v-R} \bar{M}_{X}^{T+\Delta}(R+i v)}{(R+i v)(R+i v-1)} d v
$$

where $\bar{M}_{X}^{T+\Delta}(\cdot)$ is the moment generating function of $X$ under the $(T+$ $\Delta$ )-forward measure and $R$ is such that $\bar{M}_{X}^{T+\Delta}(R+i v)$ is finite. This moment generating function would have to be computed for each of the various forward measures, but it can be directly expressed in terms of the $Q$-characteristics of the factors: the Radon-Nikodym-derivative to change from $Q$ to $Q^{T+\Delta}$ can in fact be expresses in explicit form and it preserves the affine structure, see Corollary 10.2 in [10] (For a recent account on conditions for an absolutely continuous measure transformation to preserve the affine structure see [12]).

If $M_{X}^{T+\Delta}(\cdot)$ is the moment generating function of $X$ with $p(T, T+\Delta)$ instead of $\bar{p}(T, T+\Delta)$, then

$$
\bar{M}_{X}^{T+\Delta}(z)=M_{X}^{T+\Delta}(z) A\left(z ; \theta, \kappa, \Psi_{0}^{1}, \Psi_{0}^{3}\right)
$$

for a suitable $A\left(\cdot ; \theta, \kappa, \Psi_{0}^{1}, \Psi_{0}^{2}, \Psi_{0}^{3}\right)$, where $A\left(\cdot ; \theta, \kappa, \Psi_{0}^{1}, \Psi_{0}^{2}, \Psi_{0}^{3}\right)$ $=E^{T+\Delta}\left\{\left(M_{X}^{T+\Delta}\right)^{-1} e^{z X}\right\}$ which, given the affine nature of the factors, can be explicitly computed as a function of the parameters of the model and the initial values $\Psi_{0}^{1}, \Psi_{0}^{2}, \Psi_{0}^{3}$ of the factors. As such, this may however not suffice to derive a satisfactory adjustment factor as for FRAs. 
Acknowledgements: We are grateful to Giulio Miglietta as well as to Claudio Fontana and Zorana Grbac for very constructive comments.

\section{References}

[1] M. Bianchetti, Two Curves, One Price: Pricing \& Hedging Interest Rate Derivatives, Decoupling Forwarding and Discounting Yield Curves, arXiv:0905.2770v4 (Aug. 2012)

[2] D. Brigo, M. Morini, A. Pallavicini, Counterparty Credit Risk, Collateral and Funding with pricing cases for all asset classes. Wiley, Forthcoming, 2013.

[3] C. Chiarella, C. Nikitopoulos Sklibosios, E. Schloegl, A Markovian defaultable term structure model with state dependent volatilities, International Journal of Theoretical and Applied Finance 10 (2007), pp. 155-202.

[4] C. Chiarella, S.C. Maina, C. Nikitopoulos Sklibosios, Markovian defaultable HJM term structure models with unspanned stochastic volatility. Quantitative Finance Research Centre Research Paper no. 283 (2010), University of Technology, Sydney.

[5] J.M. Corcuera, G. Farkas, W. Schoutens, E. Valkeila. A short rate model using ambit processes. In: Malliavin Calculus and Stochastic Analysis. A Festschrift in Honor of David Nualart. Springer Proceedings in Mathematics \& Statistics Volume 34 (2013), pp. 525-553. Springer Science+Business Media, New York.

[6] S. Crépey, R. Gerboud, Z. Grbac, N. Ngor, Counterparty risk and funding: The four wings of the TVA. International Journal of Theoretical and Applied Finance (online 30 April 2013).

[7] S. Crépey, Z. Grbac and H.-N. Nguyen, A multiple-curve HJM model of interbank risk. Mathematics and Financial Economics 6(3) (2012), pp. 155-190.

[8] S. Crépey, Z. Grbac, N. Ngor, D. Skovmand, A Levy HJM multiple-curve model with application to CVA computation. Preprint 2013.

[9] R. Douady, M. Jeanblanc, A rating-based model for credit derivatives, European Investment Review 1 (2002), pp. 17-29.

[10] D. Filipović, Term Structure Models A Graduate Course, Springer Verlag (2009).

[11] D. Filipović, A.B. Trolle, The term structure of interbank risk. Journal of Financial Econometrics 109 (2013), pp. 707-733. 
[12] C. Fontana, J.M. Montes, A unified approach to pricing and risk management of equity and credit risk, Journal of Computational and Applied Mathematics, 259(B) (2014), pp. 350-361.

[13] M. Fuji, Y. Shimada, A.Takahashi, A note on the construction of multiple swap curves with and without collateral. CARF Working Paper Series F-154, 2009.

[14] M. Fuji, Y. Shimada, A.Takahashi, A market model of interest rates with dynamic basis spreads in the presence of collateral and multiple currencies. Wilmott Magazine 54 (2011), pp 61-73.

[15] Z. Grbac, A. Papapantoleon, J. Schoenmakers, D. Skovmand, Affine LIBOR models with multiple curves: theory, examples and calibration. In preparation.

[16] M. Keller-Ressel, A. Papapantoleon, J. Teichmann, The Affine Libor Models, Mathematical Finance 23 (2013), pp 627-658.

[17] C. Kenyon, Short-Rate Pricing After the Liquidity and Credit Shocks: Including the Basis (August 18, 2010). Available at SSRN: http://ssrn.com/abstract=1558429 or http://dx.doi.org/10.2139/ssrn.1558429.

[18] M. Kijima, K.Tanaka, T. Wong, A multi-quality model of interest rates, Quantitative Finance, 9(2) (2009), pp 133-145.

[19] F. Mercurio, Interest Rates and The Credit Crunch: New Formulas and Market Models. Bloomberg Portfolio Research Paper 2010-01.

[20] F. Mercurio, LIBOR Market Models with Stochastic Basis. Bloomberg Education and Quantitative Research Paper 2010-05.

[21] A. Pallavicini, D. Brigo, Interest-Rate Modelling in Collateralized Markets: Multiple curves, credit-liquidity effects, CCPs, (2013) arXiv:1304.1397v1

[22] V. Piterbarg, Funding beyond discounting: collateral agreements and derivatives pricing. Risk Magazine, 24 (2010), pp 97-102. 